SELECTED PAPER AT NCSP'16

\title{
Synchronization State of Chaotic Circuit Containing Time Delay in One Direction
}

\author{
Seiya Kita, Yoko Uwate and Yoshifumi Nishio \\ Department of Electrical and Electronic Engineering, Tokushima University \\ 2-1 Minami-Josanjima, Tokushima 770-8506, Japan \\ E-mail: $\{$ kita, uwate, nishio\}@ee.tokushima-u.ac.jp
}

\begin{abstract}
A synchronization state can be observed in coupled circuits. Furthermore, an interesting synchronization state was confirmed in coupled time-delayed chaotic circuits. In this study, we propose novel coupled systems and investigate the synchronization state in coupled time-delayed chaotic circuits. The proposed coupling methods of time-delayed chaotic circuits depend on the attractor type. We focus on the relationships between the synchronization state and the coupling method. Moreover, we investigate the special coupling methods of time-delayed circuits in this study.
\end{abstract}

\section{Introduction}

There are many nonlinear systems containing a time delay, such as neural networks, control systems, meteorological systems, biological systems and so forth, in the natural world. Thus, the investigation of the stability in such time-delayed systems is important [1]. The generation of chaos has been reported in all self-excited oscillation systems containing a time delay [2]. Such a chaotic circuit can be easily realized by using a simple electric circuit element and can be analyzed exactly. On the other hand, there are examples of nonlinear phenomena, such as chaotic synchronization and so forth [3]. In particular, many studies on the synchronization of coupled chaotic circuits have been reported [4].

In this study, we devise coupled systems that take advantage of the features of time-delayed chaotic circuits. The novel coupled systems utilize the characteristics of circuits having time-delayed feedback. These circuits also contain gain-controlled chaotic oscillators with a time delay and a feedback system for controlling the gain. We investigate the synchronization state in coupled time-delayed chaotic circuits. By carrying out computer simulations, it is shown that the time delay of subcircuits changes the synchronization state.

\section{Time-Delayed Chaotic Circuit}

Figure 1 shows a time-delayed chaotic circuit. This circuit consists of one inductor $L$, one capacitor $C$, one linear nega- tive resistor $-g$ and one linear positive resistor $G$, whose amplitude is controlled by a switch containing a time delay. The current flowing through the inductor $L$ is $i$, and the voltage across the capacitor $C$ is $v$. The circuit equations are normalized to Eqs. (1) and (2) by changing the variables as below.

(A) In the case of a switch connected to the negative resistor,

$$
\left\{\begin{array}{l}
\dot{x}=y \\
\dot{y}=2 \alpha y-x
\end{array}\right.
$$

(B) In the case of a switch connected to the positive resistor,

$$
\left\{\begin{array}{l}
\dot{x}=y \\
\dot{y}=-2 \beta y-x
\end{array}\right.
$$

We change the parameters and variables as follows.

$$
\begin{gathered}
i=\sqrt{\frac{C}{L}} V_{t h} x, v=V_{t h} y, t=\sqrt{L C} \tau \\
g \sqrt{\frac{C}{L}}=2 \alpha \text { and } G \sqrt{\frac{C}{L}}=2 \beta
\end{gathered}
$$

The switching operation used to control the amplitude of the oscillator is shown in Fig.2. This switching operation includes a time delay denoted by $T_{d}$. First, the switch is connected to the negative resistor. Then, the voltage $v$ is amplified while it is oscillated until $v$ exceeds the threshold voltage $V_{t h}$. Second, the system memorizes the time during which $v$ exceeds the threshold voltage $V_{t h}$ as $T_{t h}$. After time $T_{d}$, switch is connected to the positive resistor for time $T_{t h}$. A

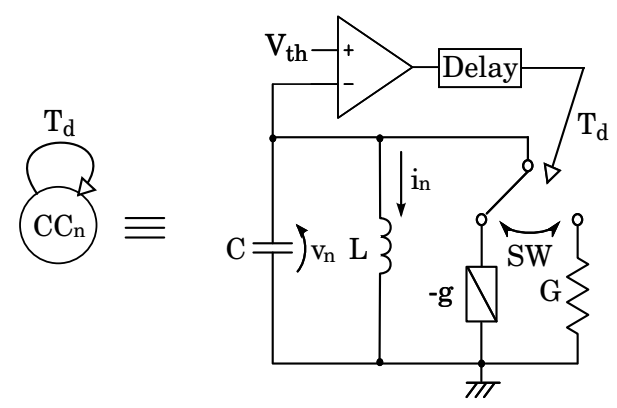

Figure 1: Time-delayed chaotic circuit 


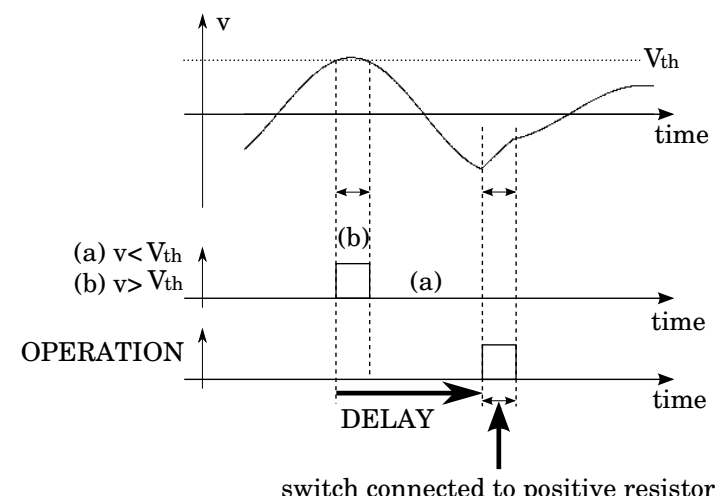

Figure 2: Switching operation

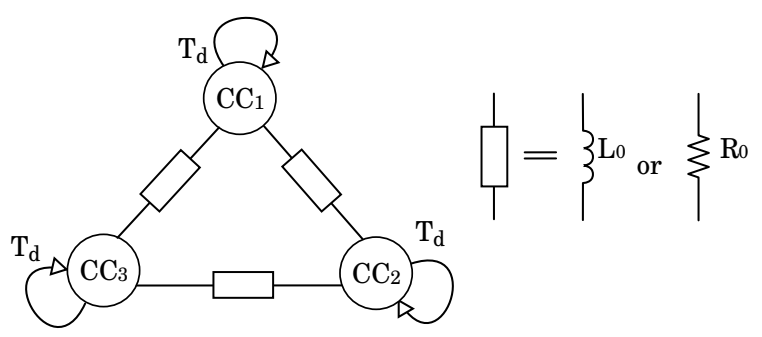

Figure 3: Ring system coupled by resistors

set of switching operations is used to control the amplitude of $v$. By applying a mapping method to this circuit, we can derive the one-dimensionale Poincare map explicitly from each circuit, and the Poincare map was proved to have a positive Lyapunov number by computer simulation [3].

\section{Coupled Time-Delayed Chaotic Ring Circuit}

In this section, we investigate the synchronization state for three time-delayed chaotic circuits coupled by different methods. Figure 3 shows a schematic of the three coupled timedelayed chaotic circuits. Two cases of interest are considered: the coupling elements are resistors $R_{0}$ and inductors $L_{0}$. We

change the parameters and variables as follows.

$$
\begin{gathered}
i_{n}=\sqrt{\frac{C}{L}} V_{t h} x_{n}, v_{n}=V_{t h} y_{n}, t=\sqrt{L C} \tau \\
g \sqrt{\frac{C}{L}}=2 \alpha, G \sqrt{\frac{C}{L}}=2 \beta \text { and } \gamma=R_{0} \sqrt{\frac{C}{L}}
\end{gathered}
$$

\subsection{Coupled by resistors $R_{0}$}

Here we discuss case of a system coupled by resistors $R_{0}$. The normalized circuit equations of the system are given as follows:

(A) In the case that the switch is connected to the negative resistor

$$
\left\{\begin{array}{l}
\dot{x}_{n}=y_{n} \\
\dot{y}_{n}=-x_{n}+2 \alpha y_{n}+\gamma\left(y_{n-1}-2 y_{n}+y_{n+1}\right)
\end{array}\right.
$$

(B) In the case that the switch is connected to the positive resistor

$$
\left\{\begin{array}{l}
\dot{x}_{n}=y_{n} \\
\dot{y}_{n}=-x_{n}-2 \beta y_{n}+\gamma\left(y_{n-1}-2 y_{n}+y_{n+1}\right)
\end{array}\right.
$$

where $n=1,2,3$ and $x_{0}=x_{3}, x_{4}=x_{1}$. Figure 4 shows some simulation results, in which the in-phase synchronization state can be observed. When the coupling strength $\gamma$ is large, full in-phase synchronization can be observed. However, full in-phase synchronization cannot be observed or synchronization is lost in the case of a small $\gamma$.

\subsection{Coupled by inductors $L_{0}$}

Figure 5 shows some simulation results in the case of timedelayed chaotic circuits coupled by inductors $L_{0}$. We change the parameters and variables when the coupled ring system is connected by inductors as follows.

$$
\begin{gathered}
i_{n}=\sqrt{\frac{C}{L}} V_{t h} x_{n}, v_{n}=V_{t h} y_{n}, t=\sqrt{L C} \tau \\
g \sqrt{\frac{C}{L}}=2 \alpha, G \sqrt{\frac{C}{L}}=2 \beta \text { and } \gamma^{\prime}=\frac{L}{L_{0}}
\end{gathered}
$$

The normalized circuit equations of the system are given as follows:

(A) In the case that the switch is connected to the negative resistor

$\left\{\begin{array}{l}\dot{x}_{n}=y_{n} \\ \dot{y}_{n}=-x_{n}+2 \alpha y_{n}+\gamma^{\prime}\left(x_{n-1}-2 x_{n}+x_{n+1}\right)\end{array}\right.$

(B) In the case that the switch is connected to the positive resistor

$\left\{\dot{x}_{n}=y_{n}\right.$

$\left\{\dot{y}_{n}=-x_{n}-2 \beta y_{n}+\gamma^{\prime}\left(x_{n-1}-2 x_{n}+x_{n+1}\right)\right.$

where $n=1,2,3$ and $x_{0}=x_{3}, x_{4}=x_{1}$. In-phase synchronization and three-phase synchronization can be observed in the ring system coupled by inductors $L_{0}$. When the coupling strength $\gamma^{\prime}$ is equal to 0.01 , synchronization is lost. Consequently, a certain coupling strength is required for synchronization.

\section{System Including Time Delay in One Direction}

The circuits in this study employ characteristic time delay methods. We have devised the coupled system shown in Fig.6. This system is coupled by resistors $R_{0}$ or inductors $L_{0}$. This system includes a time delay in one direction. 

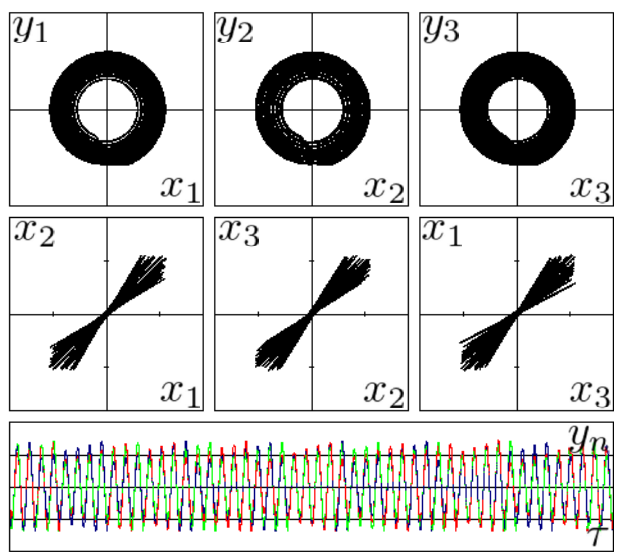

(a) $\alpha=0.015, \beta=0.5, \gamma=0.01$ and $T_{d}=\pi$

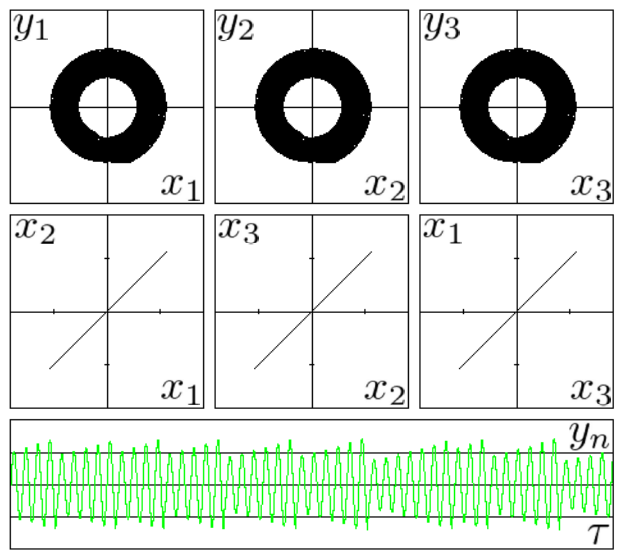

(b) $\alpha=0.015, \beta=0.5, \gamma=0.1$ and $T_{d}=\pi$

Figure 4: Simulation results of ring system coupled by resistors

\subsection{Coupled by resistors $R_{0}$}

First, we use resistors $R_{0}$ as coupling elements. The normalized circuit equations of this system are the same as Eqs.(3) and (4). The result shown in Fig.7 can be obtained from the difference in the coupling strength $\gamma$. The time waveform in Fig.7(a) shows in-phase synchronization and the amplitude of $y_{n}$ is switched sequentially. However, when $\gamma$ is larger than 0.1 , the switching synchronization state is lost and a full in-phase synchronization state can be observed.

\subsection{Coupled by inductors $L_{0}$}

When we use inductors $L_{0}$ as coupling elements, the result shown in Fig. 8 can be obtained from the difference in the coupling strength $\gamma^{\prime}$, where the normalized circuit equations are Eqs.(5) and (6). The time waveform in Fig.8(a) shows a phase difference and the amplitude of $y_{n}$ is switched sequentially. However, when $\gamma^{\prime}$ is larger than 0.1 , the switching synchronization state is lost. Generally, switching synchronization can be observed when the system including a time delay in

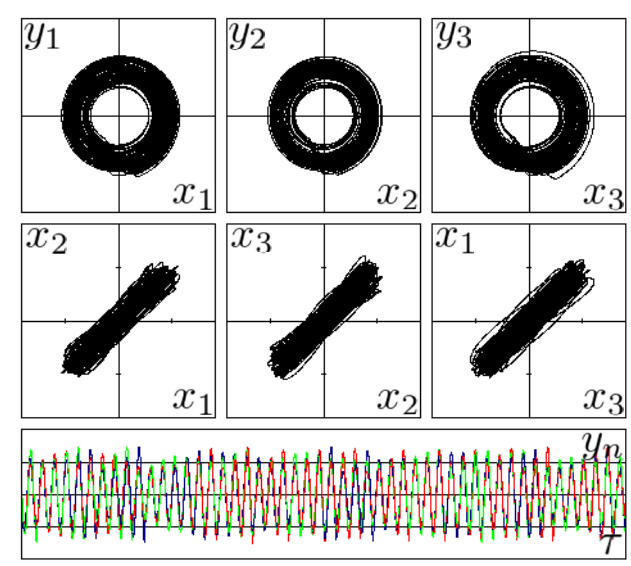

(a) $\alpha=0.015, \beta=0.5, \gamma^{\prime}=0.1$ and $T_{d}=\pi$

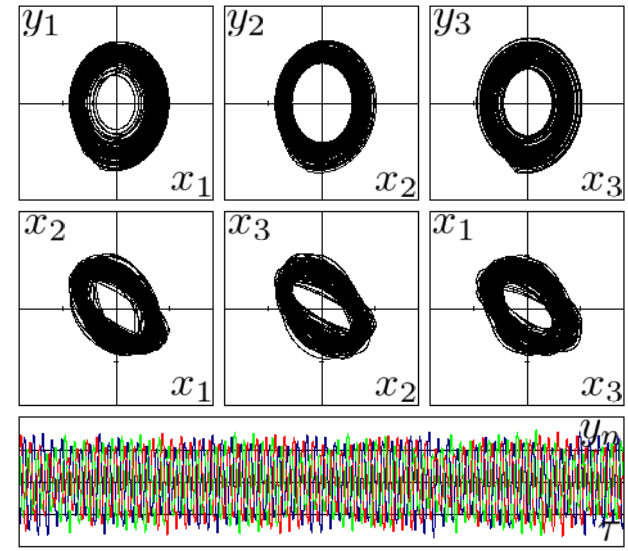

(b) $\alpha=0.015, \beta=0.5, \gamma^{\prime}=0.2$ and $T_{d}=\pi$

Figure 5: Simulation results of ring system coupled by inductors

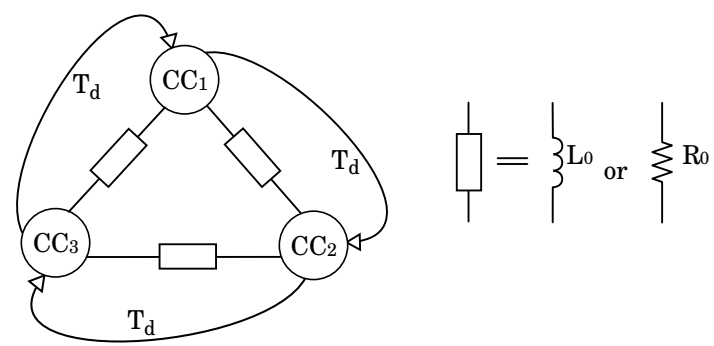

Figure 6: System including time delay in one direction

one direction is coupled by resistors $R_{0}$ or inductors $L_{0}$. The amplitude alternately diverges and converges with different divergence and convergence times.

\section{Conclusions}

In this study, we investigated the synchronization state of novel coupled systems of time-delayed chaotic ring circuits 

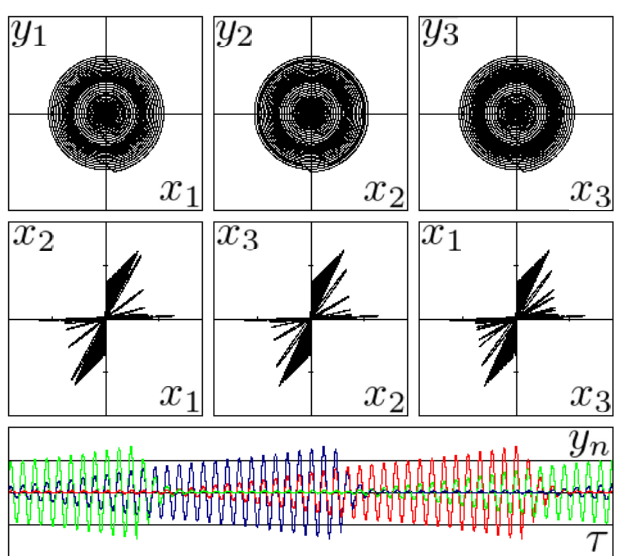

(a) $\alpha=0.015, \beta=0.5, \gamma=0.01$ and $T_{d}=\pi$

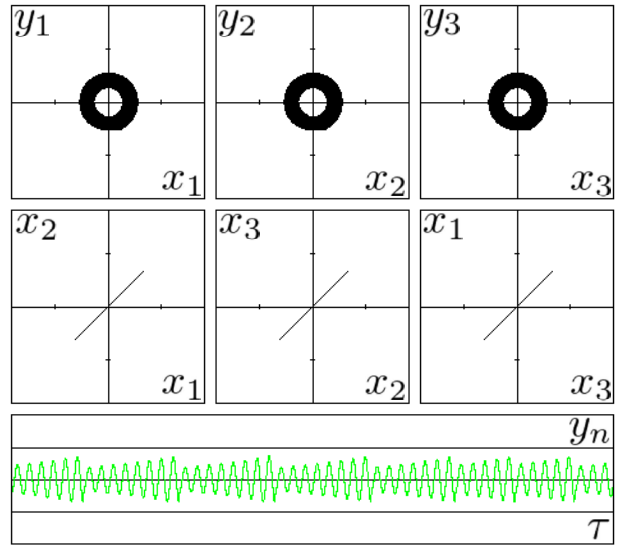

(b) $\alpha=0.015, \beta=0.5, \gamma=0.1$ and $T_{d}=\pi$

Figure 7: Simulation results of system coupled by resistors including time delay in one direction

coupled by various methods. Four types of coupling system were investigated. In the case of a ring circuit coupled by resistors, we observed an in-phase synchronization state. In the other cases of the ring circuit coupled by inductors, in-phase synchronization and three-phase synchronization states were observed. We devised coupled systems that take advantage of the features of the time-delayed chaotic circuits. As a result, some special synchronization states were observed. Switching of the amplitude of the voltage in addition to the in-phase synchronization and three-phase synchronization state were observed from the difference of coupling strength and coupling elements.

\section{Acknowledgment}

This work was partly supported by JSPS Grant-in-Aid for Challenging Exploratory Research 26540127.

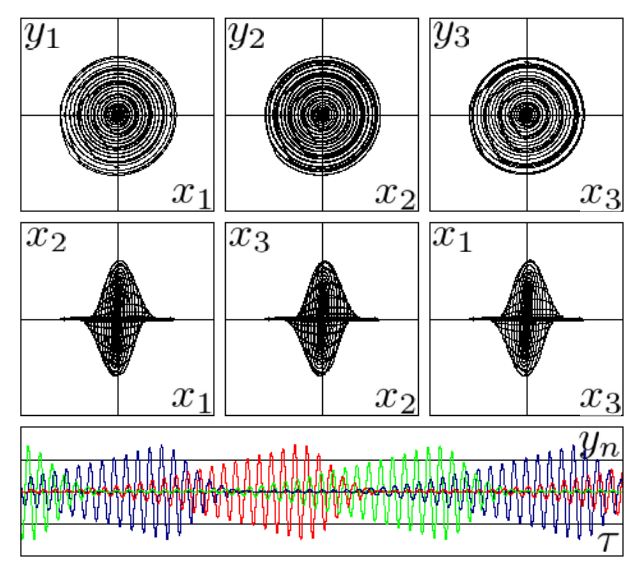

(a) $\alpha=0.015, \beta=0.5, \gamma^{\prime}=0.01$ and $T_{d}=\pi$

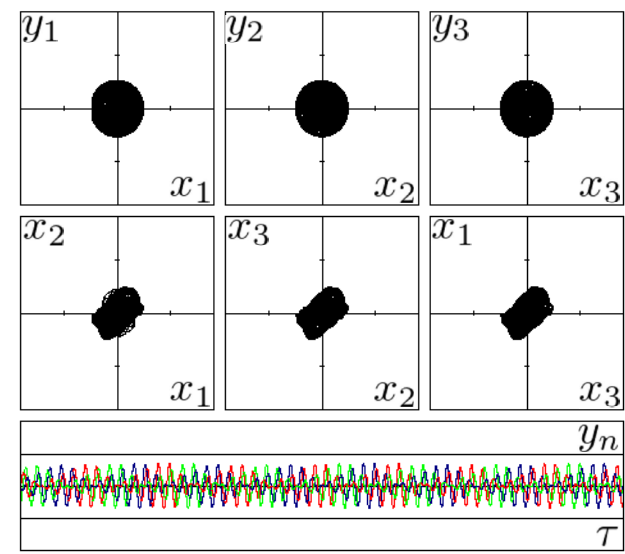

(b) $\alpha=0.015, \beta=0.5, \gamma^{\prime}=0.1$ and $T_{d}=\pi$

Figure 8: Simulation results of system coupled by inductors that combines switching including time delay in one direction

\section{References}

[1] X. Liu: Stability of impulsive control systems with time delay, Mathematical and Computer Modelling, Vol. 39, pp. 511-519, 2004.

[2] T. Maruyama, N. Inaba, Y. Nishio and S. Mori: Chaos in an auto gain controlled oscillator containing time delay, Trans. IEICE, Vol. J 72-A, pp. 1814-1820, 1989.

[3] T. Maruyama, N. Inaba, Y. Nishio and S. Mori: Chaos in self oscillator circuit containing time delay, Proceedings of IEEE Midwest Symposium on Circuits and Systems (MWSCAS'90), Vol. 2, pp. 1055-1058, 1990.

[4] L. M. Pecora and T. L. Carroll: Synchronization in chaotic systems, Physical Review Letters, Vol. 64, No. 8, pp. 821-824, 1990. 